\title{
Rheological Characterization and Constitutive Modeling of Two LDPE Melts
}

\author{
Víctor Hugo Rolón-Garrido ${ }^{\mathrm{a}}$, Radek Pivokonsky ${ }^{\mathrm{b}, \mathrm{c}}$, Petr Filip ${ }^{\mathrm{b}}$, \\ Martin Zatloukal ${ }^{\mathrm{c}}$ and Manfred H. Wagner ${ }^{\mathrm{a}}$ \\ ${ }^{a}$ Chair of Polymer Engineering/Polymer Physics, Berlin Institute of Technology (TU Berlin), \\ Fasanenstrasse 90, D-10623 Berlin, Germany \\ ${ }^{b}$ Institute of Hydrodynamics, Academy of Sciences of the Czech Republic, Pod Patankou 5, 16612 \\ Prague 6, Czech Republic \\ ${ }^{c}$ Polymer Centre, Faculty of Technology, Tomas Bata University in Zlin, TGM 275, Zlin 76272, Czech \\ Republic
}

\begin{abstract}
Experimental data of two low-density polyethylene (LDPE) melts at $200^{\circ} \mathrm{C}$ for both shear flow (transient and steady shear viscosity as well as steady first normal stress coefficient) and elongational flow (transient and steady-state elongational viscosity) as published by Pivokonsky et al. [1] were analyzed by use of the Molecular Stress Function (MSF) model for broadly distributed, randomly branched molecular structures. For quantitative modeling of melt rheology in both types of flow and in a very wide range of deformation rates, only three nonlinear viscoelastic material parameters are needed: While the rotational parameter, $a_{2}$, and the structural parameter, $\beta$, are found to be equal for the two melts considered, the melts differ in the parameter $f_{\max }^{2}$ describing maximum stretch of the polymer chains.
\end{abstract}

Keywords: Rheology, MSF model, Shear flow, Elongational flow, Strain hardening, Low-density polyethylene, Polymer melts.

PACS: 83.10.Gr, 83.10.Kn, 83.60.Df, 83.60.Df, 83.80.Sg, 83.85.Rx, 83.85.Jn, 83.85.Lq,

83.60.Fg

\section{INTRODUCTION}

To solve complex flow problems in polymer processing, a quantitative model of melt rheology, i.e. a constitutive equation is needed. A suitable constitutive model should be able to describe different types of polymer melts and different types of flow with a minimum number of material parameters.

A constitutive equation which has been used successfully to model different types of polymers under a variety of experimental flow conditions is the so-called Molecular Stress Function (MSF) model [2]. This integral model is based on the tube model of Doi and Edwards [3,4], where a polymer chain is enclosed in a tubular region delimited by the neighbouring macromolecules, but overcomes the limitations of the Doi-Edwards model by allowing not only for chain orientation, but also for chain stretch. Chain stretch is considered as a consequence of tube contraction by deformation, an idea originally proposed by Marrucci and Hermans [5]. 
More recent developments of the MSF model for linear and branched monodisperse polystyrene melts [6-8] have shown that for modeling of monodisperse melts an application of only one nonlinear parameter is sufficient. This parameter, the so-called tube diameter relaxation time $\tau_{a}$, represents the relaxation of the topological constraint caused by many surrounding chains. However, as so far no simple generalization of this modelling approach to the case of polydisperse melts is hitherto known, we will use here an earlier version of the MSF model, which has been used successfully for quantitative description of the nonlinear rheology of polydisperse linear [9] and longchain branched polymer melts $[10,11]$, polymer blends $[12,13]$ and even polymer networks [14]. Predictions have been obtained for a variety of deformations like uniaxial, equibiaxial and planar extensional flows [15] as well as for steady [2], large amplitude oscillatory [16], and also exponential shear flows [17]. Rasmussen and Yu [18] modeled quantitatively the burst of inflated cylinders of LDPE melt and obtained excellent agreement with experimental evidence. To describe polydisperse branched systems, this model requires three nonlinear parameters, which are related to the architecture of the molecules under study [10, 19].

Polymers produced at industrial scale are generally polydisperse, and LDPE, due to its radical polymerization process [20] possesses a randomly branched topology. Constitutive equations of the differential type have been used to model rheology of such complex topologies in different types of deformations (see [21-23]). Pivokonsky et al. [1] presented experimental data in shear and elongation of two highly branched film-blowing grades of LDPE, and analyzed their data successfully by use of three different advanced differential constitutive equations, the so-called XPP model, the PTT-XPP model and the modified Leonov model. It is well known that the severe drawback of constitutive equations of the differential type inheres in a large number of nonlinear parameters needed to get quantitative agreement between predictions and experimental data. Usually, two or even three different nonlinear parameters are needed for each linear-viscoelastic relaxation mode, which, e.g., for ten relaxation modes leads to 20-30 nonlinear material parameters.

Due to the completeness of the data set obtained by Pivokonsky et al. [1] and the very broad experimental window comprising many decades of deformation rates, it is the objective of the present contribution to demonstrate the modelling capabilities of the MSF model in shear and elongational flow, using the same three nonlinear material parameters for all relaxation modes.

\section{EXPERIMENTAL DATA}

The experimental data analyzed are those of Pivokonsky et al. [1]. The rheology of two highly branched LDPE materials widely used in the film blowing industry (LDPE Escorene LD165BW1, Exxon, USA, and LDPE Bralen RB0323, Slovnaft, Slovakia) was investigated. The experiments were performed at $200^{\circ} \mathrm{C}$. For both materials, the linear viscoelastic storage and loss moduli ( $G^{\prime}$ and $G^{\prime \prime}$ ), the transient and steady-state shear viscosity and first normal stress coefficient were measured with the Advanced Rheometric Expansion System (ARES 2000, Rheometrics). The capillary rheometer RH7 (Rosand Precision Ltd.) was used to 
obtain steady-state shear data and first normal stress coefficients at high shear rates. The first normal stress coefficient data were obtained by a method based on the determination of the exit pressure for a slit die. The uniaxial extensional viscosity was measured with the ARES 2000 rheometer equipped with the SER Universal Testing Platform (SER-HV-A01 model) from Xpansion Instruments [24-26]. For more detailed description of the experiments see Pivokonsky et al. [1].

From $G$ ' and $G$ ', data in [1], discrete relaxation spectra with partial moduli $g_{i}$ and relaxation times $\lambda_{i}$ were obtained and are presented in Table 1 for easy reference. Table 1 also includes the molecular characteristics of the melts investigated. For both samples, the linear viscoelastic data together with the predictions using the discrete relaxation spectra of Table 1 are presented in Figure 1 . It is obvious that the linear viscoelastic response of the two melts is very similar in the experimental window investigated. No significant influence of the different values of polydispersity is observed. Differences are seen only at low frequencies, and in agreement with the lower average molar weight of LDPE Bralen, smaller values of $G$ ' and $G$ ', are seen for Bralen in a comparison with Escorene.

\section{THEORY}

The MSF model $[2,10,27]$ is a single tube segment model. The tube diameter $a$ is assumed to be independent of the orientation of tube segments, and to decrease from its equilibrium value $a_{0}$ to a value $a$ with increasing deformation.

TABLE 1. Relaxation spectra of LDPE melts at $200^{\circ} \mathrm{C}$ [1].

\begin{tabular}{|c|c|c|c|}
\hline \multicolumn{2}{|c|}{ LDPE Escorene } & \multicolumn{2}{|c|}{ LDPE Bralen } \\
\hline \multicolumn{2}{|c|}{$M w=366300 \mathrm{~g} / \mathrm{mol}$} & \multicolumn{2}{|c|}{$M w=262000 \mathrm{~g} / \mathrm{mol}$} \\
\hline \multicolumn{2}{|c|}{$M w / M n=12.1$} & \multicolumn{2}{|c|}{$M w / M n=8.19$} \\
\hline \multirow{2}{*}{\multicolumn{2}{|c|}{$\begin{array}{c}J_{e}^{0}=1.8658 \times 10^{-3} \mathrm{~Pa}^{-1} \\
\eta_{0}=7.7103 \times 10^{4} \mathrm{~Pa} \cdot \mathrm{s}\end{array}$}} & \multirow{2}{*}{\multicolumn{2}{|c|}{$\begin{aligned} J_{e}^{0} & =9.5525 \times 10^{-4} \mathrm{~Pa}^{-1} \\
\eta_{0} & =6.2319 \times 10^{4} \mathrm{~Pa} \cdot \mathrm{s}\end{aligned}$}} \\
\hline & & & \\
\hline$g_{i}(\mathrm{~Pa})$ & $\lambda_{i}\left(\mathrm{~s}^{-1}\right)$ & $g_{i}(\mathrm{~Pa})$ & $\lambda_{i}\left(\mathrm{~s}^{-1}\right)$ \\
\hline $1.0943 \times 10^{5}$ & $1.54 \times 10^{-3}$ & $1.2144 \times 10^{5}$ & $1.34 \times 10^{-3}$ \\
\hline $3.73526 \times 10^{4}$ & $6.33 \times 10^{-3}$ & $3.52922 \times 10^{4}$ & $5.2 \times 10^{-3}$ \\
\hline $3.24093 \times 10^{4}$ & $2.602 \times 10^{-2}$ & $3.34429 \times 10^{4}$ & $2.015 \times 10^{-2}$ \\
\hline $1.52512 \times 10^{4}$ & $1.0686 \times 10^{-1}$ & $1.94803 \times 10^{4}$ & $7.804 \times 10^{-2}$ \\
\hline $1.10812 \times 10^{4}$ & $4.3891 \times 10^{-1}$ & $1.19234 \times 10^{4}$ & $3.023 \times 10^{-1}$ \\
\hline $4.83509 \times 10^{3}$ & $1.80281 \times 10^{0}$ & $5.76363 \times 10^{3}$ & $1.17104 \times 10^{0}$ \\
\hline $1.98665 \times 10^{3}$ & $7.40488 \times 10^{0}$ & $2.57463 \times 10^{3}$ & $4.53626 \times 10^{0}$ \\
\hline $4.9469 \times 10^{2}$ & $3.04149 \times 10^{1}$ & $8.00865 \times 10^{2}$ & $1.75722 \times 10^{1}$ \\
\hline $1.10156 \times 10^{2}$ & $1.24927 \times 10^{2}$ & $2.13412 \times 10^{2}$ & $6.80695 \times 10^{1}$ \\
\hline $3.33765 \times 10^{1}$ & $5.13127 \times 10^{2}$ & $3.46864 \times 10^{1}$ & $2.63681 \times 10^{2}$ \\
\hline
\end{tabular}




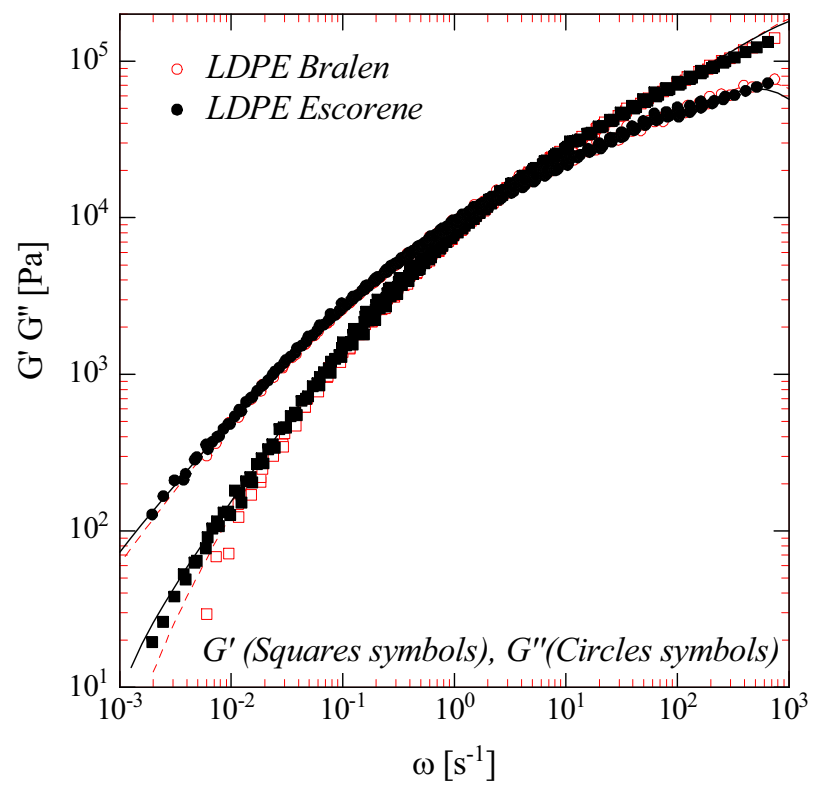

FIGURE 1. Linear viscoelastic data for LDPE Bralen (open symbols) and Escorene (closed smbols) at $200^{\circ} \mathrm{C}$. Dashed lines for LDPE Bralen and solid lines for LDPE Escorene indicate fit by use of discrete relaxation spectrum of Table 1.

The extra stress tensor $\underline{\sigma}(t)$ of the MSF model is given by a history integral of the form

$$
\underline{\underline{\sigma}}(t)=\int_{-\infty}^{t} m\left(t-t^{\prime}\right) f^{2} \underline{\underline{S}}_{D E}^{I A}\left(t, t^{\prime}\right) d t^{\prime}
$$

The strain measure $\underset{=}{S_{D E}^{I A}}$ represents the contribution to the extra stress tensor originated from the affine rotation of the tube segments assuming "Independent Alignment (IA)" [3], and is given by

$$
\underline{S}_{D E}^{I A}\left(\mathrm{t}, \mathrm{t}^{\prime}\right) \equiv 5\left\langle\frac{\underline{u}^{\prime}}{u^{\prime 2}}\right\rangle_{\mathrm{o}}=5 \underset{\underline{S}}{\underline{u^{\prime}}}\left(t, t^{\prime}\right)
$$

with $\underline{\underline{S}}=\underline{\underline{S}}\left(t, t^{\prime}\right)$ being the relative second order orientation tensor. $\underline{u}^{\prime} \underline{u}^{\prime}$ is the dyad of a deformed unit vector $\underline{u}^{\prime}=\underline{u}^{\prime}\left(t, t^{\prime}\right)$,

$$
\underline{u^{\prime}}=\underline{\underline{F}}_{t}^{-1} \cdot \underline{u}
$$


$\underline{\underline{F}}_{t}^{-1}=\underline{\underline{F}}_{t}^{-1}\left(t, t^{\prime}\right)$ is the relative deformation gradient tensor, and $u^{\prime}$ represents the length of $\underline{u}^{\prime}$. The orientation average is indicated by $<\ldots>_{0}$,

$$
\langle\ldots\rangle_{\mathrm{o}} \equiv \frac{1}{4 \pi} \oiint[\ldots] \sin \theta_{o} d \theta_{o} d \varphi_{o}
$$

i.e. an average over an isotropic distribution of unit vectors $\underline{u}$.

The square of the relative tension in the chain segment, $f^{2}$, is related to the strain energy stored in the polymeric system, and is determined as a solution of an evolution equation derived from an energy balance argument [2]. Long-chain branched polymer melts do not only exhibit a higher level of strain-hardening, but also a steeper slope of the elongational viscosity after inception of strain-hardening [9, 28]. This effect has been modeled by considering a strain energy function based on the idea that while the backbone of the branched molecule is stretched by deformation, side chains are compressed [10, 29]. It has been shown later that this consideration can be, with no significant difference, replaced by the assumption that the side chains are oriented (but not stretched) with the flow [19].

For polydisperse branched random polymer melts, considering the change of free energy and the constraint release (CR) term, the evolution equation for $f^{2}$ was found to be [10]

$$
\frac{\partial f^{2}}{\partial t}=\frac{\beta f^{2}}{1+\frac{\beta-1}{f^{4}}}\left[(\underline{\underline{\kappa}}: \underline{\underline{S}})-\frac{C R}{f^{2}-1}\right]
$$

with

$$
C R=a_{1}\left(f^{2}-1\right)^{2} \sqrt{\underline{D}^{2}: \underline{S}}+a_{2}\left(f^{2}-1\right)^{2} \sqrt{|\underline{W} \underline{D}: \underline{S}|}
$$

where $\underline{\underline{\kappa}}, \underline{\underline{D}}$ and $\underline{\underline{W}}$ are the velocity gradient, deformation rate and vorticity tensors, respectively. In compliance with the model, the nonlinear parameter $\beta$ is directly related to the molecular structure of the polymer, and represents the ratio of the molar mass of the (branched) polymer chain to the molar mass of the backbone alone. For linear polymers, it therefore takes the value of 1 [10]. In elongation experiments, $\beta$ determines the slope of the time-dependent elongational viscosity after the inception of strain hardening [10]. The nonlinear parameters $a_{1}$ and $a_{2}$ verify $a_{1} \geq 0$ and

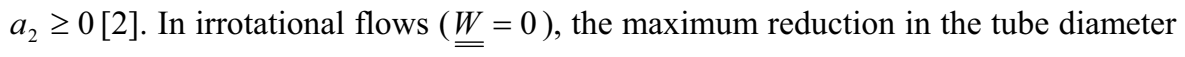
corresponding to the maximum stretch of chain segments $f=f_{\max }$ is reached when $\partial f^{2} / \partial t=0$. Hence the factor $a_{1}$ can be expressed as a function of $f_{\max }^{2}$ as

$$
a_{1}=\frac{1}{f_{\max }^{2}-1}
$$


The parameter $f_{\max }^{2}$ represents the maximum strain energy which can be stored in the polymeric system in nonlinear deformation [13], and determines the steady-state value of the viscosity in extensional flows [2]. In the Gaussian approximation used here, relative tension and relative stretch are proportional to each other. Therefore $f_{\max }$ represents also the maximum stretch that polymer chains can be subjected to due to flow before chains slip past one another without any further stretch. A correlation between $f_{\max }^{2}$ and coil contraction due to branching was found by Rolón-Garrido and Wagner [19], with $f_{\max }^{2}$ increasing with increasing coil contraction. The parameter $a_{2}$ governs the additional dissipation process in shear flow due to its rotational character.

\section{RESULTS AND DISCUSSION}

The nonlinear parameters $f_{\max }^{2}$ and $\beta$ were obtained by fitting predictions of the MSF model to the transient elongational data and, keeping these parameters fixed, the transient shear data were modeled. The parameter $a_{2}$, which influences shear predictions only, was found to attain a value of $a_{2}=0.036$ in agreement with earlier results of Wagner et al. [2] for LDPE. The nonlinear model parameters are summarized in Table 2 .

The experimental elongation rates investigated were the same for both polymers, with the exception of the three lowest values, which were applied only to LDPE Escorene. Therefore, a direct comparison of experimental data and predictions in elongational flow are presented in Figure 2 for both melts simultaneously. The strain hardening of LDPE Bralen is seen to be clearly more pronounced than the one of Escorene. With the exception of the lowest elongational rate in the case of Escorene, and the transients at the highest elongation rates, possibly due to experimental problems like finite rise-time effects, the agreement between experimental data and predictions is remarkably good. It is well known and confirmed in Figure 2 that the higher the value of the strain rate, the faster the positive deviation with respect to the linear-viscoelastic start-up occurs, i.e. strain hardening is strain dependent.

As the MSF model used is time-deformation separable, this demonstrates that the data are time-deformation separable in the experimental window considered, i.e. for elongation rates from at least 0.01 up to $20 \mathrm{~s}^{-1}$.

LDPE is produced industrially by two different types of reactor, tubular and autoclave reactors [30]. The type of reactor determines the type of branching structure: the autoclave process leads to a higher long-chain branching (LCB) content than the tubular process [31], and therefore to a more globular structure for LDPE produced in autoclave reactors than the more extended coil configuration of LDPE produced in tubular reactors [32].

TABLE 2. Nonlinear parameters of MSF model

\begin{tabular}{lccc}
\hline Sample & $\boldsymbol{f}_{\boldsymbol{M A X}}^{2}$ & $\boldsymbol{\beta}$ & $\boldsymbol{a}_{2}$ \\
\hline LDPE-Bralen & 150 & 2 & 0.036 \\
LDPE-Escorene & 70 & 2 & 0.036 \\
\hline
\end{tabular}




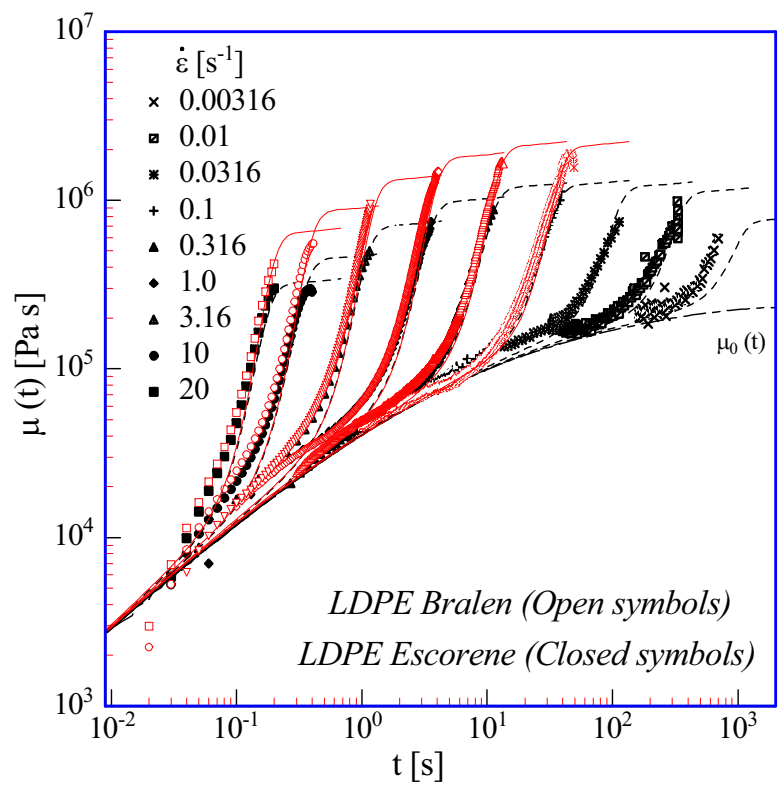

FIGURE 2. Comparison between experimentally determined transient uniaxial elongational viscosity data and predictions of MSF model (lines) at $200^{\circ} \mathrm{C}$. Dashed dotted lines represent the linear viscoelastic limit.

By analyzing elongational data of several LDPEs, it was found that LDPE produced by tubular reactors could be modelled quantitatively using $\beta=2$, while the quantitative predictions for LDPE produced by autoclave reactors required values between $\beta=2$ and $\beta=4$ [10]. For the two LDPE melts considered here, predictions using $\beta=2$ gave the best agreement with experimental data. As the nonlinear parameter $a_{2}$, which was found to be optimal for the shear flow simulations presented below, is the same for both types of LDPE, the only nonlinear parameter differentiating between the two LDPE melts is $f_{\max }^{2}$. We find $f_{\max }^{2}=70$ for Escorene, and $f_{\max }^{2}=150$ for Bralen, in agreement with the higher strain-hardening capability of the latter and the fact that Bralen is produced by the autoclave process resulting in a more globular structure. According to the interpretation of the parameter $f_{\max }^{2}$, LDPE Bralen can store more elastic strain energy due to enhanced chain stretching than Escorene, possibly due to a smaller relative size of the polymer coil [19]. Figure 3 presents the comparison of experimental data and predictions of the MSF model for the transient shear viscosity. 

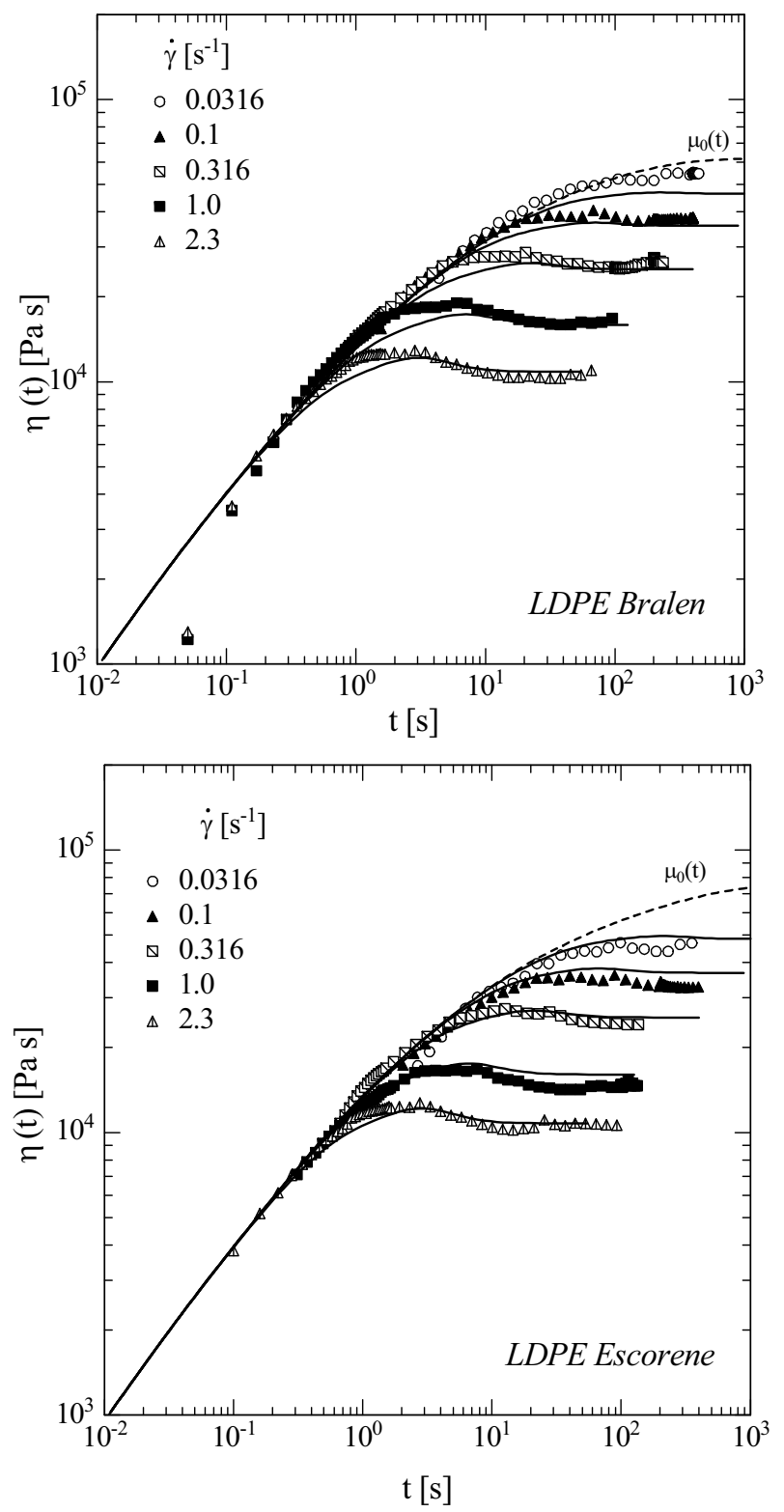

FIGURE 3. Comparison between experimentally determined transient shear viscosity data and predictions of MSF model (lines) at $200^{\circ} \mathrm{C}$. Dashed dotted line represents the linear viscoelastic limit.

The agreement is nearly quantitative; the slight deviations observed at the start-up are due to finite rise time effects of the rheometer. The steady-state shear viscosities of both LDPE melts are presented in Figure 4. The steady-state shear viscosities obtained 
by rotational rheometry at shear rates less than $2 \mathrm{~s}^{-1}$ are predicted quantitatively, while the capillary rheometer data (Figure 4) are slightly lower than both the rotational data in the shear rate range of the overlap, and the predictions. Nevertheless, the agreement is remarkable considering the shear-rate range covered. It can also be observed from the experiments as well as from the predictions that in contrast to the elongational behavior, the difference between the shear rheology of both samples is negligible at shear rates above $0.03 \mathrm{~s}^{-1}$. This is due to the fact that in shear flow, much less chain stretching is occurring, and thus the difference in the chain stretching capabilities of the two melts is not detectable in shear flow. The difference in the predictions at shear rates below $0.03 \mathrm{~s}^{-1}$ is due to the different molecular weights of the polymers.

In Figure 4 also the comparison between the steady-state elongational viscosities measured and predicted is presented. For the steady-state elongational viscosities measured we follow [1] in taking the maximum values of the transient elongational viscosities experimentally obtained. It is clear that not for all elongation rates the true steady-state elongational viscosity has been reached experimentally, and therefore the determination of $f_{\max }^{2}$ relies on the "bending-over" of the elongational viscosity at higher strains, i.e. on the transition behavior to steady state.

Nevertheless, it is clear that LDPE Bralen features a higher steady-state elongational viscosity in the experimentally observed window than Escorene, due to the fact that molecular chains of Bralen are more stretched than those of Escorene.

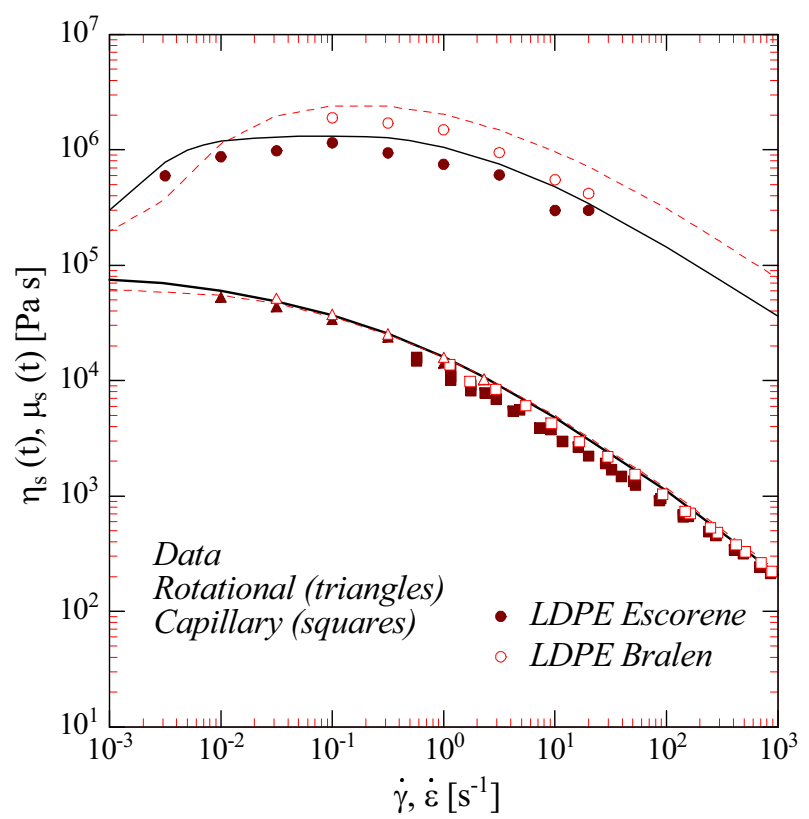

FIGURE 4. Comparison between experimentally determined steady shear and uniaxial elongational viscosities data and predictions of the MSF model (lines) at $200^{\circ} \mathrm{C}$. 
Furthermore, in contrast to shear flow, the difference in the strain hardening capabilities of the two melts persists at higher elongation rates, confirming the higher sensitivity of the extensional deformation to differences in molecular topology.

The high polydispersity of the samples is responsible for the broad transition of the steady elongational viscosities to a power-law behavior at higher deformation rates, with a slope corresponding to the slope of the shear viscosity at higher shear rates.

The experimentally determined transient first normal stress coefficient is a measurement particularly difficult to perform [1]. Nevertheless, agreement between model and data is fair at low shear rates, while at higher shear rates, predictions are below experimental data. This is confirmed in Figure 5, where for both melts the steady-state first normal stress coefficient measured by rotational rheometry atnlower shear rates and obtained by exit pressure measurements in slit die rheometry at higher shear rates is contrasted with the predictions of the model. Deviations between experimental data and predictions decrease at higher shear rates. As in the case of the shear viscosity, there is no appreciable distinction in the first normal stress differences between the two melts both experimentally determined and modeled, reflecting again the limited sensitivity of nonlinear shear flow with respect to the structure of the polymer.

It should be emphasized that the agreement between experimental data and the MSF model for both shear and elongational flow and for a very wide range of deformation rates is achieved by use of only three nonlinear material parameters.

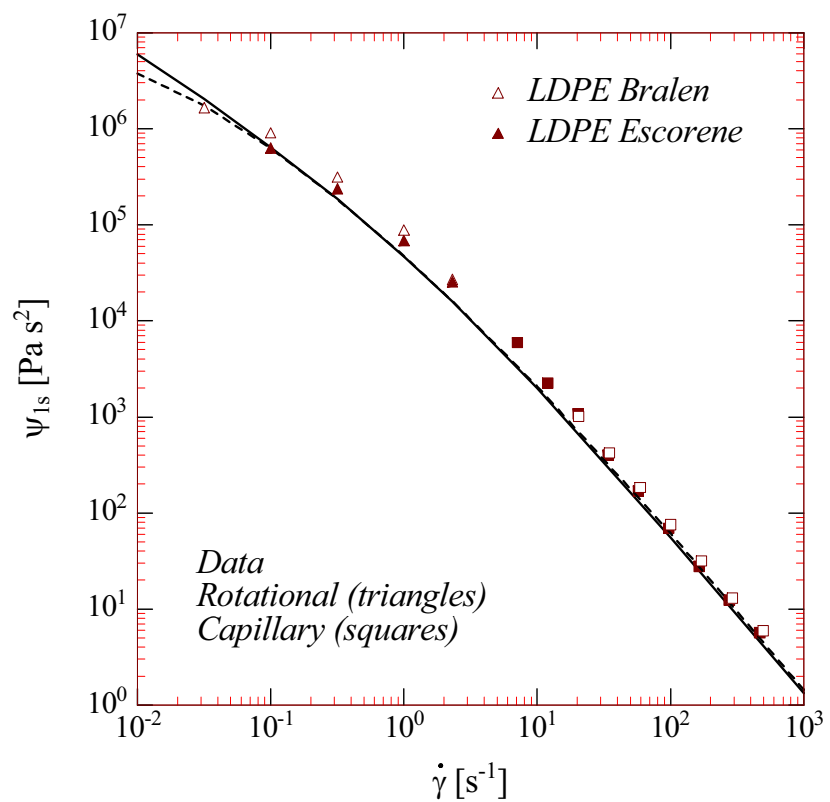

FIGURE 5. Comparison between experimentally determined steady state first normal stress coefficient data and predictions of the MSF model (lines) at $200^{\circ} \mathrm{C}$. 


\section{CONCLUSION}

Experimental data of two low-density polyethylene (LDPE) melts at $200^{\circ} \mathrm{C}$ for both shear flow (transient and steady shear viscosity as well as transient and steady first normal stress coefficient) and elongational flow (transient and steady elongational viscosity) as published in [1] were analyzed by use of the Molecular Stress Function (MSF) model for broadly distributed, randomly branched molecular structures. The data set allowed the MSF model for the first time to be tested for LDPE in shear and elongation simultaneously and covering a very wide range of deformation rates. In contrast to differential constitutive equations, where typically a different set of nonlinear parameters is needed for each linear-viscoelastic mode of the relaxation spectrum to achieve agreement between data and model, the MSF model needs only three nonlinear material parameters.

Since the rotational parameter, $a_{2}$, and the structural parameter, $\beta$, are equal for the two LDPE melts considered here, the difference in the rheological behavior can be expressed by a single parameter, the maximum chain stretch $f_{\max }$. As $f_{\max }$ of LDPE Bralen is higher than $f_{\max }$ of Escorene, it may be concluded that Bralen has a higher long-chain branching content and a more compact coil structure than Escorene, and therefore is able to store more elastic energy in elongational deformation than Escorene. While the two melts show a considerable difference in the amount of strain hardening in elongational flow, their shear flow behavior features only minor differences, confirming the higher sensitivity of extensional deformation to the topology of polymer melts.

\section{ACKNOWLEDGEMENTS}

V.H.R.G. was COFAA fellow. Part of this work was supported by SIP-IPN (20082971), México, by Grant Agency of the Czech Republic (Grant No. A200600703), Ministry of Education of the Czech Republic (MSM 7088352101), as well as by the German Science Foundation (DFG).

\section{REFERENCES}

1. R. Pivokonsky, M. Zatloukal and P. Filip, J. Non-Newtonian Fluid Mech. 135, 58-67 (2006).

2. M. H. Wagner, P. Rubio and H. Bastian, J. Rheol. 45, 1387-1412 (2001).

3. M. Doi and S.F. Edwards, Trans. Faraday Soc II 74, 1802-1817 (1978).

4. M. Doi and S.F. Edwards, Trans. Faraday Soc II 75, 38-54 (1979).

5. G. Marrucci and J.J. Hermans, Macromolecules 13, 380-387 (1980).

6. M. H. Wagner, S. Kheirandish, and O. Hassager, J. Rheol. 49, 1317-1327 (2005).

7. V.H. Rolón-Garrido, M.H. Wagner, C. Luap, T. Schweizer, J. Rheol. 50, 327-340 (2006).

8. M.H. Wagner and V.H. Rolón-Garrido, J. Rheol. 52 1049-1068 (2008).

9. M.H. Wagner, P. Ehrecke, P. Hachmann and J. Meissner, J. Rheol. 42, 621-638 (1998).

10. M.H. Wagner, M. Yamaguchi, M. Takahashi, J. Rheol. 47, 779-793 (2003).

11. A.D. Gotsis, B.L.F. Zeevenhoven and C. Tsenoglou, J. Rheol. 48, 895-914 (2004).

12. M.H. Wagner, S. Kheirandish and M. Yamaguchi, Rheol. Acta 44, 198-218 (2005).

13. M.H. Wagner, S. Kheirandish, K. Koyama, A. Nishioka, A. Minegishi, T. Takahashi, Rheol. Acta 44, 235-243 (2005). 
14. M.H. Wagner and J. Schaeffer, J. Rheol. 37, 643-661 (2003).

15. M.H. Wagner, H. Bastian, P. Hachmann, J. Meissner, S. Kurzbeck, H. Münstedt and F. Langouche, Rheol. Acta 39, 97-109 (2000).

16. P. Wapperom, A. Leygue and R. Keunings, J. Non-Newt. Fluid Mech. 130, 63-76 (2005).

17. M.H. Wagner, V.H. Rolón-Garrido, C.K. Chai, Rheol. Acta 45, 164-173 (2005).

18. H.K. Rasmussen and K Yu, Rheol. Acta 47, 149-157 (2008).

19. V.H. Rolón-Garrido, M.H. Wagner, Rheol Acta 46, 583-593 (2007).

20. G. Odian, Principles of Polymerization, John Wiley and Sons, USA, 1981.

21. N.J. Inkson, T.C.B. McLeish, O.G. Harlen and D.J. Groves, J. Rheol. 43, 873-896 (1999).

22. W.M.H. Verbeeten, G.W.M. Peters and F.P.T. Baaijens, J. Rheol. 45, 823-843 (2001).

23. N. Clemeur, R.P.G. Rutgers, B. Debbaut, Rheol. Acta 42, 217-231 (2003).

24. M.L. Sentmanat, US Patent No. 6,578,413 (17 June 2003).

25. M.L. Sentmanat, Rheol. Acta 43, 657-669 (2004).

26. M.L. Sentmanat, B.N. Wang and G.H. McKinley, J. Rheol. 49, 585-606 (2005).

27. M.H. Wagner and J. Schaeffer, J. Rheol. 36, 1-26 (1992).

28. M.H. Wagner, Korea-Australia Rheology Journal 11, 293-304 (1999).

29. M.H. Wagner, J. Hepperle and H. Münstedt, J. Rheol. 48, 489-503 (2004).

30. L. Marini, C. Georgakis, AIChE 30, 401-408 (1984).

31. G. Gianotti, A. Cicuta and D. Romanini, Polymer 21, 1087-1091 (1980).

32. R. Kuhn, H. Krömer and G. Roßmanith, Ang. Makrom. Chem. 40/41, 361-389 (1974). 
Copyright of AIP Conference Proceedings is the property of American Institute of Physics and its content may not be copied or emailed to multiple sites or posted to a listserv without the copyright holder's express written permission. However, users may print, download, or email articles for individual use. 